\title{
Sodbrennen
}

\section{Ein Symptom, zwei verschiedene Krankheiten: Das Infomobil klärt auf}

_über sieben Wochen lang war das Infomobil der „Initiative Gesunder Magen“ in ganz Deutschland unterwegs, um über das unangenehme Brennen hinter dem Brustbein aufzuklären - Sodbrennen. Zum vierten Mal in Folge tourte der

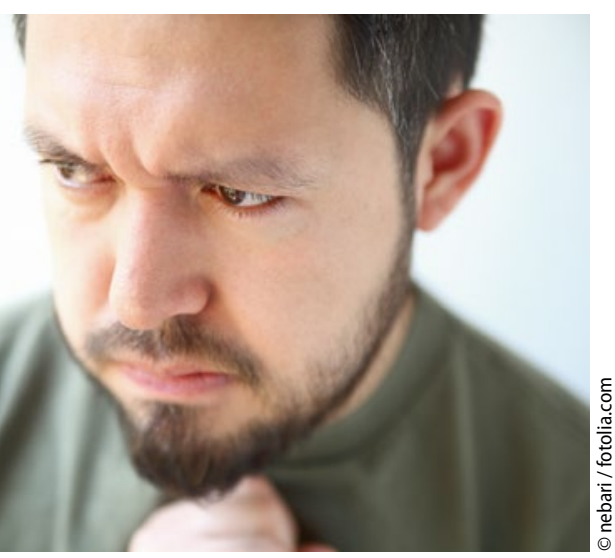

Sodbrennen - Refluxkrankheit oder funktionelle Dyspepsie?
Bus mit Informationen durch 36 Städte. Viele Besucher nutzten auch in diesem Jahr die Gelegenheit, sich über das Thema Sodbrennen zu informieren. Denn obwohl Sodbrennen ein echtes Volksleiden ist, wissen viele Menschen nicht, dass dahinter zwei verschiedene Krankheiten stecken können.

Ziel der Initiative ist es unter anderem, den Menschen zu erklären, dass Sodbrennen oft nicht „zu viel Säure“ bedeuten kann, sondern häufig auch „Säure am falschen Ort“. „Haben Sie nur Sodbrennen - oder auch andere Magenbeschwerden?" - Diese Frage kann entscheidend sein für die richtige Diagnosestellung und damit auf für eine erfolgreiche Therapie.

\section{Alle Magenbeschwerden abfragen}

Für die richtige Diagnosestellung ist es wichtig, alle Magenbeschwerden zu nen- nen bzw. abzufragen. Wird das unangenehme Brennen hinter dem Brustbein als Einzelsymptom genannt, liegt die Diagnose Refluxkrankheit nahe. Kommen weitere Magenbeschwerden wie Übelkeit, Völlegefühl oder Magenschmerzen hinzu, kann dies auf eine funktionelle Dyspepsie hindeuten.

Diesen Patienten kann Iberogast ${ }^{\circledR}$ aufgrund seiner Multi-Target-Wirkung schnell und effektiv helfen. Es ist deshalb ein Mittel der Wahl. Protonenpumpeninhibitoren (PPI), die für die funktionelle Dyspepsie nicht zugelassen sind, sind hier oft nur unzureichend wirksam.

Informationen zu der Aufklärungskampagne finden Interessierte unter www.initiative-gesunder-magen.de -

Red.

- Nach Informationen von Steigerwald

\section{Schlaganfallpråvention bei Vorhofflimmern}

\section{Hohe Adhärenz unter Antikoagulation mit Apixaban}

_ Patienten mit nicht-valvulärem Vorhofflimmern haben ein fünffach höheres Risiko für einen Schlaganfall als Personen ohne diese Herzrhythmusstörung. Für die Behandlung mit neuen oralen Antikoagulanzien stehen bislang nur wenige Daten zur Adhärenz und Persistenz zur Verfügung.

\section{Prüfung im Praxisalltag}

AEGEAN (Assessment of an Education and Guidance program for Eliquis ${ }^{\circledR}$ Adherence in Non-valvular atrial fibrillation) ist die erste prospektive, randomisierte klinische Studie zu Apixaban (Eliquis ${ }^{\circledR}$ ), die Adhärenz und Persistenz bei einem begleitenden Schulungsprogramm im Vergleich zur Standardaufklärung ohne Schulungsprogramm untersucht. Bei Apixaban handelt es sich um einen neuen oralen, reversiblen, hochselektiven, direkten Faktor-Xa-In- hibitor. „In die europäische MulticenterStudie wurden Patienten mit nicht-valvulärem Vorhofflimmern oder Vorhofflattern eingeschlossen“, berichtete Prof. Georg Gahn, Direktor der Neurologischen Klinik am Städtischen Klinikum Karlsruhe.

\section{Spezielle Schulungsprogramme nicht unbedingt erforderlich}

583 Patienten erhielten bei zweimal täglicher Apixaban-Gabe die Standardaufklärung. 579 Patienten absolvierten darüber hinaus ein Schulungsprogramm, das eine zusätzliche Patientenbroschüre, verschiedene Erinnerungshilfen für die Tabletteneinnahme und die Möglichkeit zur Kontaktaufnahme mit einer „virtuellen Klinik“ umfasste. Die Adhärenz wurde mithilfe eines elektronischen „Helping Hand“-Gerätes gemessen.
Die Adhärenz lag nach 24 Wochen in der Patientengruppe mit Standardaufklärung bei $88,5 \%$ und in der Gruppe mit dem zusätzlichen Schulungsprogramm bei $88,3 \%(p=0,89)$. Die Persistenz nach 24 Wochen lag in der Gruppe mit Standardaufklärung bei $90,5 \%$ und in der Gruppe mit begleitendem Schulungsprogramm bei $91,1 \%(\mathrm{p}=0,76)$.

„Dies bedeutet, dass die Patienten eine hohe Adhärenz und Persistenz unter Apixaban aufwiesen, die durch ein zusätzliches Schulungsprogramm nicht beeinflusst wurde“, resümierte Gahn. Die Ergebnisse nach 48 Wochen werden für Ende 2016 erwartet.

\section{Gudrun Girrbach}

- Symposium „Schlaganfallprävention bei Vorhofflimmern Warum sollten wir uns die Antikoagulation zu Herzen nehmen?", 88. Kongresses der Deutschen Gesellschaft für Neurologie (DGN); Düsseldorf, September 2015 (Veranstalter: Bristol-Myers Squibb/Pfizer) 